\title{
Controlled-Release Oxycodone and Pregabalin in the Treatment of Neuropathic Pain: Results of a Multicenter Italian Study
}

\author{
Antonio Gatti ${ }^{a}$ Alessandro Fabrizio Sabato ${ }^{a}$ Roberto Occhioni ${ }^{b}$ \\ Gianni Colini Baldeschic ${ }^{c}$ Carlo Reale $^{d}$

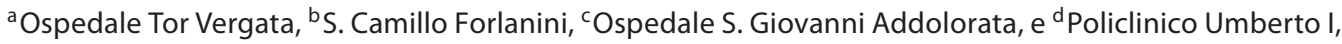 \\ Roma, Italia
}

\section{Key Words}

Neuropathic pain $\cdot$ Oxycodone $\cdot$ Pregabalin •

Combination therapy

\begin{abstract}
Aims: The aim of our study was to compare the efficacy, safety, and quality of life of combination therapy with controlledrelease (CR) oxycodone plus pregabalin versus monotherapy with either $\mathrm{CR}$ oxycodone or pregabalin in patients with neuropathic pain. Materials and Methods: Patients with moderate to severe neuropathic pain, despite the use of various pharmacologic treatments prior to study entry, were enrolled $(n=409)$ and treated with CR oxycodone plus pregabalin ( $n=169)$, CR oxycodone $(n=106)$, and pregabalin $(n=134)$. Pain intensity was rated on an 11-point numerical rating scale (NRS). Results: The combination of CR oxycodone plus pregabalin and CR oxycodone monotherapy were both more effective for alleviating neuropathic pain than pregabalin monotherapy (reduction in NRS value: 80,76 , and $46 \%$, respectively; $p \leq 0.003$ ). Significantly greater improvements from baseline in quality of life were reported with combination therapy than with monotherapy $(p=0.0009)$. At the end of treatment, the majority (91.2\%) of patients receiving $\mathrm{CR}$ oxycodone plus pregabalin found that the treatment had been 'effective' or 'very effective'. Combination therapy also allowed a dose reduction of both agents (22\% for $\mathrm{CR}$ oxycodone and $51 \%$ for pregabalin) compared with
\end{abstract}

the dosages of the respective monotherapies. Combination therapy had a superior safety profile compared with pregabalin monotherapy. Conclusions: The combination of $C R$ oxycodone plus pregabalin may represent a valuable addition to the existing pharmacotherapy for neuropathic pain and warrants further investigation.

Copyright $\odot 2008$ S. Karger AG, Basel

\section{Introduction}

Neuropathic pain is caused by nerve injury. Due to the heterogeneity of etiologies and the complexity of the underlying mechanisms, the treatment of painful neuropathies can be challenging $[1,2]$. Current treatment for neuropathic pain includes antidepressants such as tricyclic agents and selective serotonin reuptake inhibitors, anticonvulsant agents (e.g. gabapentin, pregabalin), topical lidocaine, and oral opioids (e.g. morphine, oxycodone) [3, 4]. International guidelines do not specifically recommend a single first-line treatment $[3,4]$, and clinical experience shows that at least $30 \%$ of patients do not achieve pain relief with monotherapy [5].

Since the response to monotherapy remains unsatisfactory for a significant proportion of patients, combination therapy is common practice in the management of painful neuropathies. In addition, the combination of drugs with different mechanisms of action can be expect-

\section{KARGER}

Fax +4161306 1234 E-Mail karger@karger.ch www.karger.com (c) 2008 S. Karger AG, Basel

0014-3022/09/0613-0129\$26.00/0

Accessible online at:

www.karger.com/ene
Antonio Gatti

Azienda Universitaria Policlinico Tor Vergata

Dipartimento AFA Servizio di Anestesia e Terapia del Dolore

Viale Oxford 81, IT-00100 Rome (Italy)

Tel. +39062090 0638, E-Mail antonio.gatti@ptvonline.it 
ed to improve clinical efficacy and safety $[1,6]$. The combination of opioid analgesics and calcium channel modulators has shown promising results in both animal models and in one clinical study evaluating the combination of morphine and gabapentin $[7,8]$. Further investigation is required to understand the potential of this combination of agents.

In light of these findings, we set out to investigate the efficacy of combination therapy based on pregabalin, a novel voltage-gated calcium channel modulator, and controlled-release (CR) oxycodone, a strong opioid, in a population of patients with neuropathic pain refractory to various treatments including combination therapies.

Pregabalin has been extensively characterized in terms of its efficacy and safety and is currently one of the recommended first-line treatments for neuropathic pain $[3$, 4]. Its efficacy has been demonstrated in a number of randomized controlled trials in patients with neuropathic pain of various etiologies [9-12]. The introduction of a long-acting formulation of oxycodone has significantly improved the management of chronic pain requiring continuous treatment [13]. Oral CR oxycodone can be administered with longer dosing intervals and its sustained analgesic effect on chronic pain, including neuropathic pain, has been demonstrated in clinical studies [14-17]. Despite this evidence and the general consensus among experts that careful use of opioids can be a valuable and safe approach to pain, the place of oxycodone in the treatment of painful neuropathies is still being debated, primarily due to concern regarding the development of tolerance and addiction $[13,18]$.

In Italy, opioids are considerably underused in pain management and as a result clinical experience with these analgesics is limited [19]. The aim of our study was to compare the efficacy and safety of combination therapy with CR oxycodone plus pregabalin with that of monotherapy with either drug alone in patients with neuropathic pain that has not been adequately relieved by previous treatments.

\section{Materials and Methods}

\section{Patients}

A total of 800 patients who had had uncontrolled chronic pain for $\geq 6$ months and who reported to the Pain Therapy Ward in 11 Italian centers were screened. Four hundred and nine patients (176 males, 233 females), who were at least 18 years of age, with chronic neuropathic pain were enrolled in the study. Pain intensity at recruitment varied from moderate to severe. All eligible patients had failed to respond to a variety of analgesic treatments, including opioids and multiple-drug therapy. Patients with onco- logical pain and patients who were treated unsuccessfully with pregabalin were excluded from the study.

The Research Ethics Boards at the participating centers approved the study protocol and each patient gave written informed consent before entering the study. The study was conducted in accordance with the Helsinki Declaration of 1975 as revised in 2000 .

\section{Study Medications}

Patients received oral CR oxycodone (OxyContin ${ }^{\circledR}$, Mundipharma, Italy) monotherapy, oral pregabalin (Lyrica ${ }^{\circledR}$, Pfizer, Italy) monotherapy, or the combination of CR oxycodone plus pregabalin for 90 days. The two drugs were administered according to the manufacturers' prescribing information. Patients were started on dosages included in the recommended ranges $[3,4]$, as required by their condition. The initial mean daily dose of CR oxycodone was $24.1 \mathrm{mg}$ in the monotherapy arm, and $19.4 \mathrm{mg}$ for the combination therapy arm. The initial mean daily dose of pregabalin was $85.6 \mathrm{mg}$ in the monotherapy arm and $108.1 \mathrm{mg}$ in the combination therapy arm. Daily doses were titrated at scheduled visits on days 7, 14, 21, 28, 35, 56, 75 and 90 to achieve optimal efficacy and tolerability, based on patient response and adverse events. The treatment dosages were modified based on the pain intensity recorded at visits and during the phone interview.

Patients were allowed to continue current treatment for comorbid diseases. Concomitant analgesic medication (oral morphine 5 or $10 \mathrm{mg}$ per day) was used for the management of breakthrough pain, as required.

Study Design

The study was an open-label, prospective, multicenter comparison of the efficacy, safety, and impact on quality of life (QoL) of 3 pharmacologic treatments in patients with neuropathic pain of various etiologies. Patients receiving pregabalin who had partial pain control were assigned to the pregabalin monotherapy arm for 90 days $(n=134)$; patients with pain which was not controlled by other drugs were randomized to the CR oxycodone arm $(\mathrm{n}=106)$ or the CR oxycodone and pregabalin arm for 90 days $(n=169)$. Patient numbers were uneven in these two latter treatment arms because more patients refused to take CR oxycodone monotherapy than CR oxycodone and pregabalin combination therapy. Patients in the CR oxycodone arm who refused to take opiates were excluded from the study.

Efficacy and tolerability were the primary endpoints. Pain relief was the primary measure of efficacy. Pain intensity was assessed at study entry and during the 8 follow-up visits. Patients were asked to rate pain during the past $24 \mathrm{~h}$ on a numerical rating scale (NRS; $0=$ no pain, $10=$ worst pain imaginable). A 2-point reduction was described as clinically meaningful by Farrar et al. [20] and Rowbotham [21]. The first 5 follow-up visits were once every 7 days, while the last 3 visits were scheduled every $2-3$ weeks (follow-up visits on days $7,14,21,28,35,56,75$, and 90 ; day $0=$ beginning of treatment).

Secondary endpoints were the comparison of daily dosages for monotherapy versus combination therapy at the end of treatment, impact on QoL, and patient assessment of treatment efficacy.

The interference of pain with QoL was compared for each of the 3 treatment regimens. The Brief Pain Inventory (BPI) questionnaire, completed at study entry and at each follow-up visit, was used to evaluate how everyday life was influenced by pain 


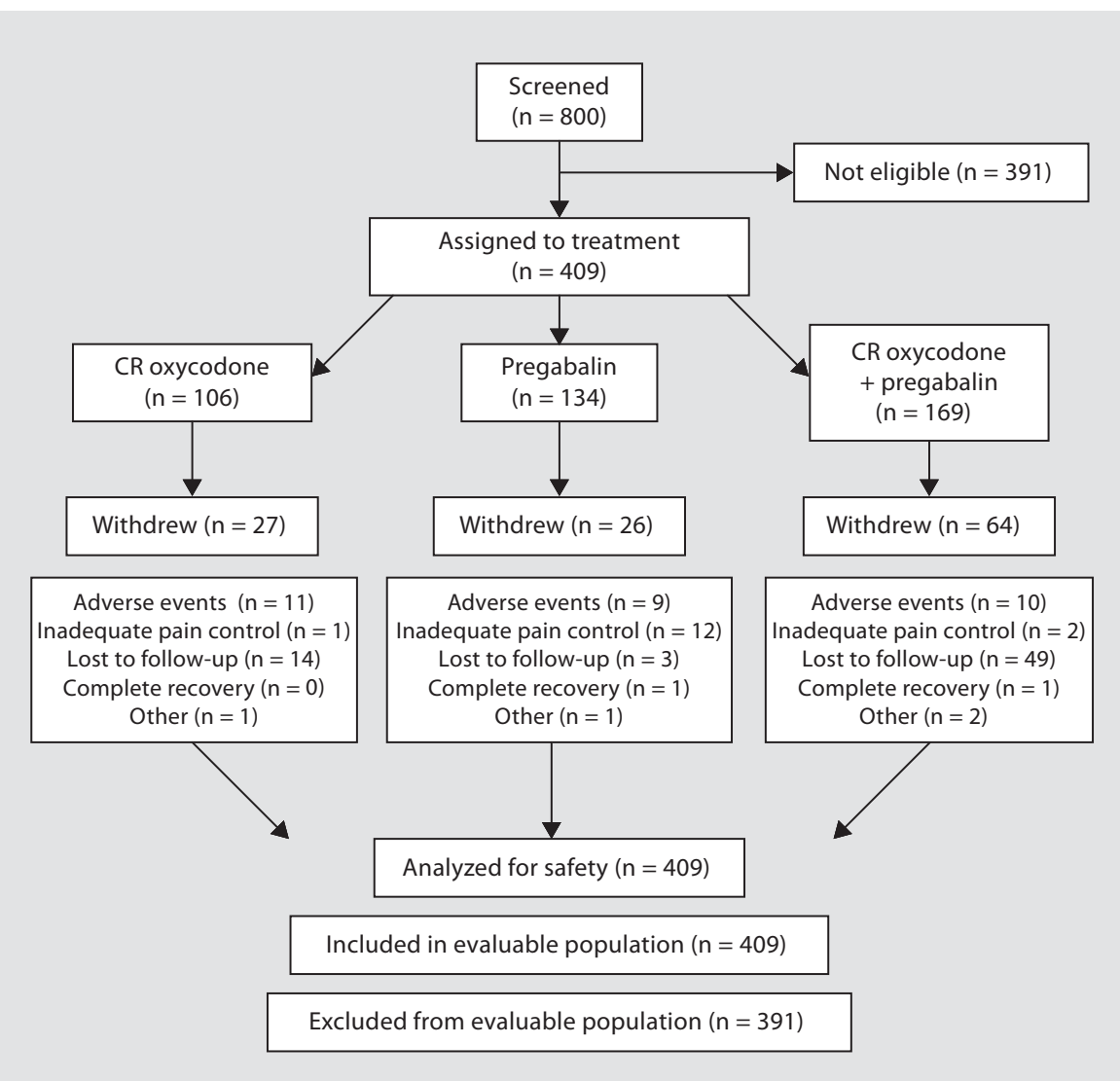

Fig. 1. Patient disposition.

during the treatment $[22,23]$. The BPI questionnaire evaluates pain intensity on an NRS ( $0=$ no pain; $10=$ worst pain imaginable) and the impact of pain on QoL by rating pain interference across seven domains (general activity, mood, walking ability, work, sleep, relation with other people, life enjoyment) on an NRS $(0=$ does not interfere; $10=$ completely interferes $)$.

For the comparison of the tolerability of each treatment, adverse events were recorded at each follow-up visit.

At each follow-up visit, patients were also asked to evaluate the effectiveness of the treatment by completing a questionnaire with the following ratings: not effective, slightly effective, effective but poorly tolerated, effective, very effective.

\section{Statistical Analysis}

In general, only descriptive summary statistics were used to analyze study results. Baseline disease characteristics were compared between treatment groups. The efficacy comparisons used analysis of covariance summary measures, which summarized the within-patient repeated measures for pain intensity through the mean of post-treatment measures utilizing this as a response variable in an analysis of covariance model where baseline pain intensity is used as a covariate to evaluate the treatment efficacy. The tolerability of the 3 regimens was based on the $95 \%$ confidence interval of the difference between treatments in the proportion of patients experiencing adverse events.

\section{Results}

\section{Patient Characteristics}

The patient disposition is shown in figure 1. All of the 409 patients enrolled were included in the evaluable patient population (the per-protocol population). Of the 169 patients on combination therapy with CR oxycodone plus pregabalin, 10 were excluded from the study because of adverse events, although they continued treatment but with adjusted dosages. Reasons for discontinuation were inadequate pain control $(n=2)$, surgery $(n=1)$, death ( $\mathrm{n}=1$; deemed unrelated to study treatment) and complete recovery $(n=1)$. In the group of 106 patients on CR oxycodone, 11 withdrew due to adverse events, 1 because pain control was inadequate, and 1 because of surgery. Details of withdrawal because of an adverse event were not recorded in the pregabalin group. Fourteen patients (13.2\%) in the CR oxycodone arm, 26 patients (19\%) in the pregabalin arm and 49 patients (29\%) receiving combination therapy were lost to follow-up. 
Fig. 2. Changes in average pain intensity during treatment with CR oxycodone plus pregabalin, CR oxycodone alone, or pregabalin alone. Pain was rated on an NRS $(0=$ no pain, $10=$ worst pain imaginable).

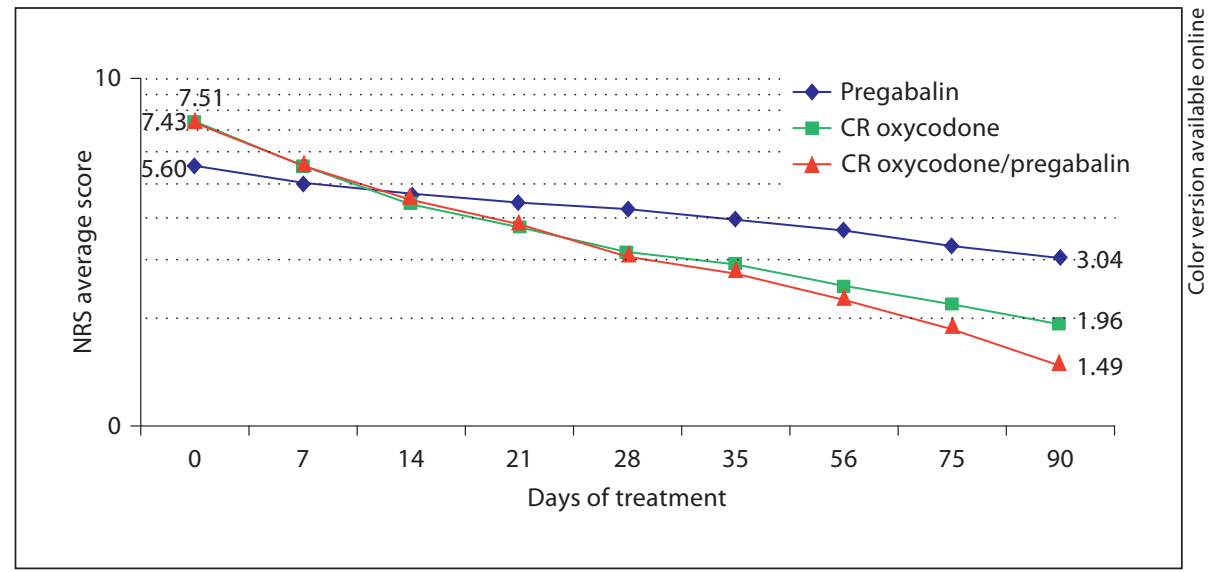

Table 1. Patient characteristics

\begin{tabular}{|c|c|c|c|}
\hline & \multicolumn{3}{|l|}{ Treatment } \\
\hline & $\begin{array}{l}\text { CR } \\
\text { oxycodone }\end{array}$ & pregabalin & $\begin{array}{l}\text { CR oxycodone } \\
+ \text { pregabalin }\end{array}$ \\
\hline Patients & 106 & 134 & 169 \\
\hline Male & 39 & 61 & 76 \\
\hline Female & 67 & 73 & 93 \\
\hline Mean age, years & $65(37-90)$ & $61(34-77)$ & $62(21-84)$ \\
\hline \multicolumn{4}{|l|}{ Underlying disease, $\%$} \\
\hline Stenosis MSC & 22.6 & 20.9 & 19.5 \\
\hline FBSS & 22.6 & 16.4 & 17.2 \\
\hline PHN & $12.3^{*}$ & 28.4 & 26.6 \\
\hline $\mathrm{DPN}$ & 17.0 & 14.9 & 16.0 \\
\hline Radiculopathy & 25.5 & 19.4 & 20.7 \\
\hline NRS, mean score & 7.5 & 5.6 & 7.4 \\
\hline \multicolumn{4}{|c|}{ Treatment before study entry, \% } \\
\hline NSAIDs & 7.6 & & 13.0 \\
\hline Weak opioids & 11.3 & & 2.4 \\
\hline Strong opioids & 3.8 & & 1.2 \\
\hline Antidepressants & 0 & & 0 \\
\hline Anticonvulsants & 1.9 & & 3.0 \\
\hline $\begin{array}{l}\text { Invasive therapy } \\
\text { (surgery) }\end{array}$ & 0.9 & & 1.2 \\
\hline Multiple-drug therapy & 73.6 & & 78.1 \\
\hline No treatment & 0.9 & & 1.2 \\
\hline
\end{tabular}

DPN = Diabetic painful neuropathy; FBSS = failed back surgery syndrome; $\mathrm{MSC}=$ medullary spinal canal; $\mathrm{PHN}=$ postherpetic neuropathy. ${ }^{*} \mathrm{p}<0.05$ vs. pregabalin, CR oxycodone + pregabalin. Figures in parentheses indicate ranges.
Patient demographics and baseline characteristics are summarized in table 1 . The underlying causes of neuropathic pain in the study population were: failed back surgery syndrome (18.3\%), postherpetic neuralgia (23.5\%), radiculopathy $(21.5 \%)$, painful diabetic neuropathy $(15.9 \%)$, and stenosis of the spinal medullary canal (20.8\%). With the exception of postherpetic neuropathy, which occurred in significantly $(\mathrm{p}<0.05)$ fewer patients in the CR oxycodone monotherapy treatment arm compared to the other 2 treatment arms, these diseases occurred with a similar incidence in the 3 treatment arms (table 1). At baseline, the mean pain intensity scores (score $>7=$ severe) were similar in the groups receiving monotherapy with CR oxycodone and combination therapy; these scores were higher than the mean score for the group receiving pregabalin monotherapy (score 5.6).

Prior to study entry, the majority of patients received pharmacologic treatment for neuropathic pain, including NSAIDs, antidepressants, and anticonvulsant agents. Potent opiates were administered in only $2.5 \%$ of cases. The majority of patients $(>70 \%)$ received multiple-drug therapies. A combination therapy was NSAIDs and mild opiates.

\section{Pain Intensity}

Combination therapy with CR oxycodone plus pregabalin and CR oxycodone monotherapy resulted in faster and more substantial pain relief than pregabalin monotherapy (fig. 2). After 90 days of treatment, the mean NRS value for the CR oxycodone plus pregabalin arm had decreased by $80 \%$ compared with baseline. In contrast, the mean NRS score for patients in the pregabalin arm had decreased by $46 \%$ ( $\mathrm{p}<0.003$ vs. combination therapy), while that of those in the CR oxycodone arm had de- 


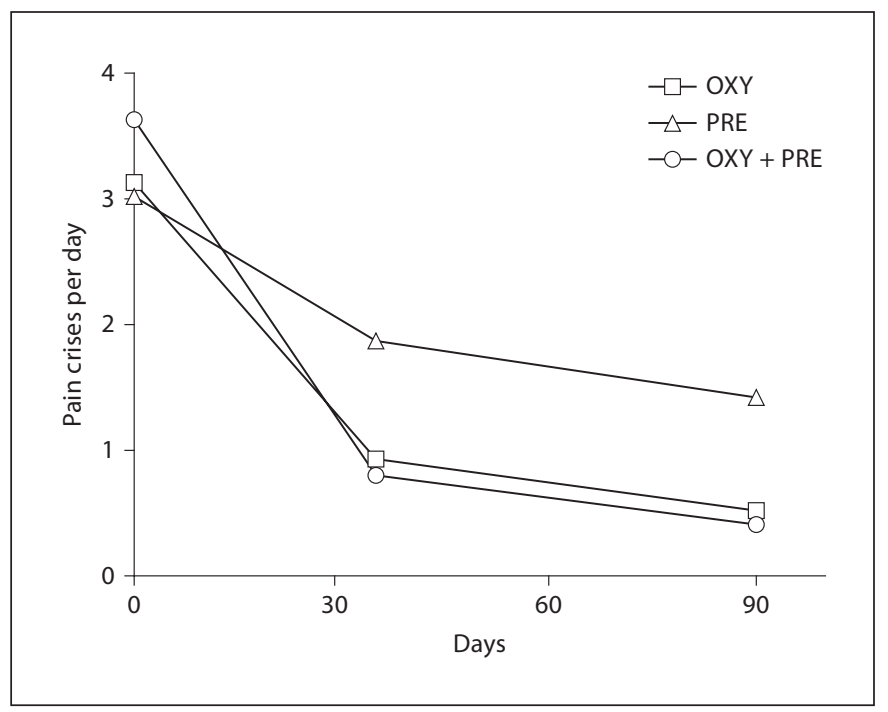

Fig. 3. Number of daily pain crisis episodes during treatment with CR oxycodone (OXY), pregabalin (PRE), or CR oxycodone plus pregabalin (OXY + PRE).

creased by $76 \%$ ( $p<0.001$ vs. combination therapy) compared with baseline.

\section{Daily Pain Crises}

At baseline, the 3 treatment arms had a similar mean number of pain crises per day (CR oxycodone plus pregabalin: 3.63; CR oxycodone: 3.13; pregabalin: 3.02). At the end of treatment, patients receiving CR oxycodone plus pregabalin and those receiving oxycodone monotherapy ( $\mathrm{p}<0.05$ vs. combination therapy), reported a substantial decrease in the mean number of pain crises per day compared with patients receiving pregabalin monotherapy ( $\mathrm{p}<0.001$ vs. combination therapy) (fig. 3 ).

\section{Mean Daily Medication Doses}

Combination treatment was effective at lower mean doses of CR oxycodone and pregabalin compared with either drug administered as monotherapy. At the end of treatment, patients receiving CR oxycodone plus pregabalin were given on average $22 \%$ less CR oxycodone and $51 \%$ less pregabalin than the respective monotherapy groups (fig. 4). In the group receiving CR oxycodone, the mean daily dose of CR oxycodone was $24.1 \mathrm{mg}$ at the beginning and $46.1 \mathrm{mg}$ at the end of the study; the mean daily doses of CR oxycodone and pregabalin in the combined treatment were 19.4 and $108.1 \mathrm{mg}$ at the beginning and 35.8 and $141.5 \mathrm{mg}$ at the end; in the group receiving

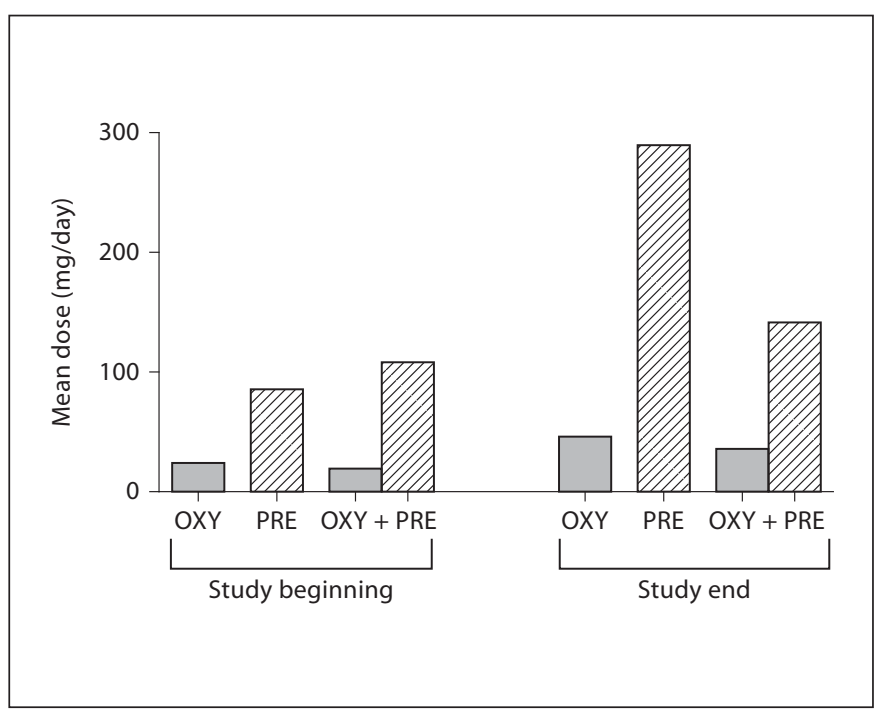

Fig. 4. Dose changes during treatment, comparing the mean doses of CR oxycodone (OXY) and pregabalin (PRE) in monotherapy or combination therapy $(\mathrm{OXY}+\mathrm{PRE})$ at study beginning and study end (90 days).

pregabalin, the mean daily dose at the beginning was 85.6 $\mathrm{mg}$ and at the end $289.5 \mathrm{mg}$.

\section{Quality of Life}

The interference of pain with activities of daily living was substantially reduced at 90 days in patients receiving CR oxycodone plus pregabalin compared with those on monotherapy. At 90 days, combination therapy was also beneficial for other QoL parameters, namely walking ability, work, and relations with other people, for which combined therapy consistently achieved lower scores than monotherapy with either drug (table 2).

The overall decrease in BPI scores at the end of treatment compared to baseline was $70.9 \%$ for combination treatment with CR oxycodone plus pregabalin $(\mathrm{p}=0.0009$ vs. both monotherapies), $60.7 \%$ for CR oxycodone monotherapy, and $42.8 \%$ for pregabalin monotherapy; fig. 5).

\section{Patient Evaluation of Efficacy}

At the end of the study, $91.2 \%$ of patients receiving combination therapy with CR oxycodone plus pregabalin and $95.6 \%$ of patients receiving CR oxycodone found the treatment 'effective' or 'very effective'. In contrast, in the group receiving pregabalin monotherapy, less than $20 \%$ of patients rated the treatment outcome positively (fig. 6). 


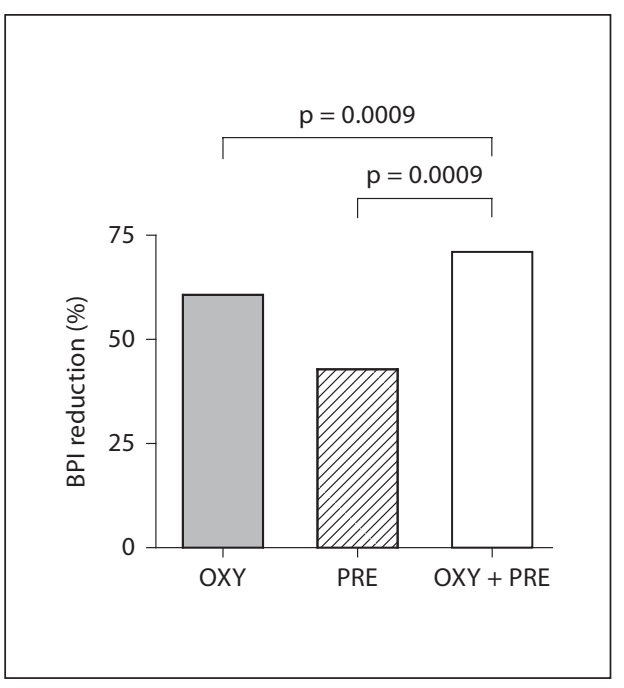

Fig. 5. The impact of treatment with CR oxycodone (OXY), pregabalin (PRE), or CR oxycodone plus pregabalin (OXY + PRE) on QoL. Percent reductions from baseline in BPI values at the end of treatment.

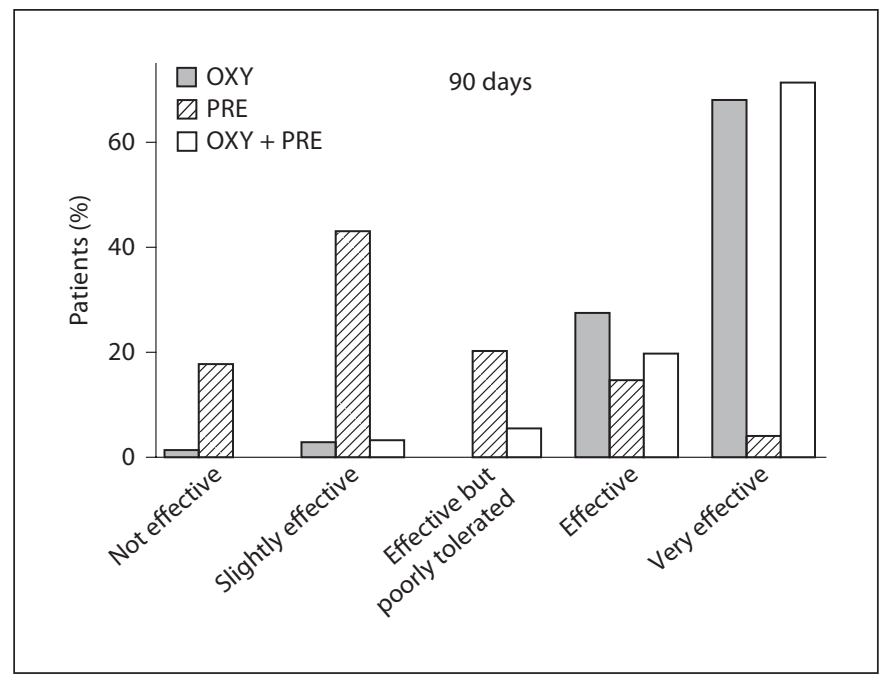

Fig. 6. Patient evaluation of treatment efficacy at 90 days for $\mathrm{CR}$ oxycodone (OXY), pregabalin (PRE) and CR oxycodone plus pregabalin (OXY + PRE).

Table 2. The impact of treatment with CR oxycodone, pregabalin, or CR oxycodone plus pregabalin on QoL: BPI ratings of pain interference with everyday life (scale $0-10 ; 0=$ does not interfere, $10=$ completely interferes)

\begin{tabular}{|c|c|c|c|c|c|c|}
\hline & \multicolumn{2}{|l|}{ Pregabalin } & \multicolumn{2}{|c|}{ CR oxycodone } & \multicolumn{2}{|c|}{ CR oxycodone + pregabalin } \\
\hline & T0 (0 days) & T8 (90 days) & T0 (0 days) & T8 (90 days) & T0 (0 days) & T8 (90 days) \\
\hline General activity & 6.33 & 3.67 & 7.43 & 2.97 & 7.80 & 2.02 \\
\hline Mood & 5.12 & 2.83 & 6.78 & 2.44 & 6.23 & 1.54 \\
\hline Walking ability & 6.04 & 3.61 & 7.03 & 2.45 & 7.77 & 2.00 \\
\hline Work & 6.15 & 3.70 & 8.01 & 3.03 & 7.46 & 2.43 \\
\hline Relations with other people & 4.18 & 2.40 & 6.23 & 2.34 & 6.78 & 1.65 \\
\hline Sleep & 4.02 & 2.29 & 6.78 & 2.65 & 6.65 & 2.22 \\
\hline Life enjoyment & 4.66 & 2.45 & 6.02 & 3.00 & 7.00 & 2.55 \\
\hline
\end{tabular}

\section{Safety Analysis}

Overall, combination therapy had a better safety profile than monotherapy with CR oxycodone and pregabalin, with a dropout rate due to adverse events of $5.9 \%$ compared to 10.4 and $19.0 \%$, respectively. The most frequently reported adverse events were somnolence and peripheral edema with pregabalin, and somnolence and constipation with CR oxycodone (fig. 7). Combination therapy was most frequently associated with constipation (fig. 7). Their frequency, however, tended to decrease over the course of the study in all treatment arms. At the end of treatment, $45.1 \%$ of patients receiving combination therapy, $42.2 \%$ of patients receiving CR oxycodone and $34.8 \%$ receiving pregabalin monotherapy reported no adverse events.

The combination of CR oxycodone and pregabalin resulted in an improved adverse event profile compared with pregabalin monotherapy (fig. 7). The incidence of peripheral edema was substantially reduced, throughout the entire study, in the group treated with the combination therapy compared with pregabalin monotherapy. Nausea and vomiting were also improved by the administration of the combination treatment compared with the two monotherapies. 
Fig. 7. Frequency of the most common adverse events in patients receiving CR oxycodone (OXY), pregabalin (PRE), or CR oxycodone plus pregabalin (OXY + PRE).

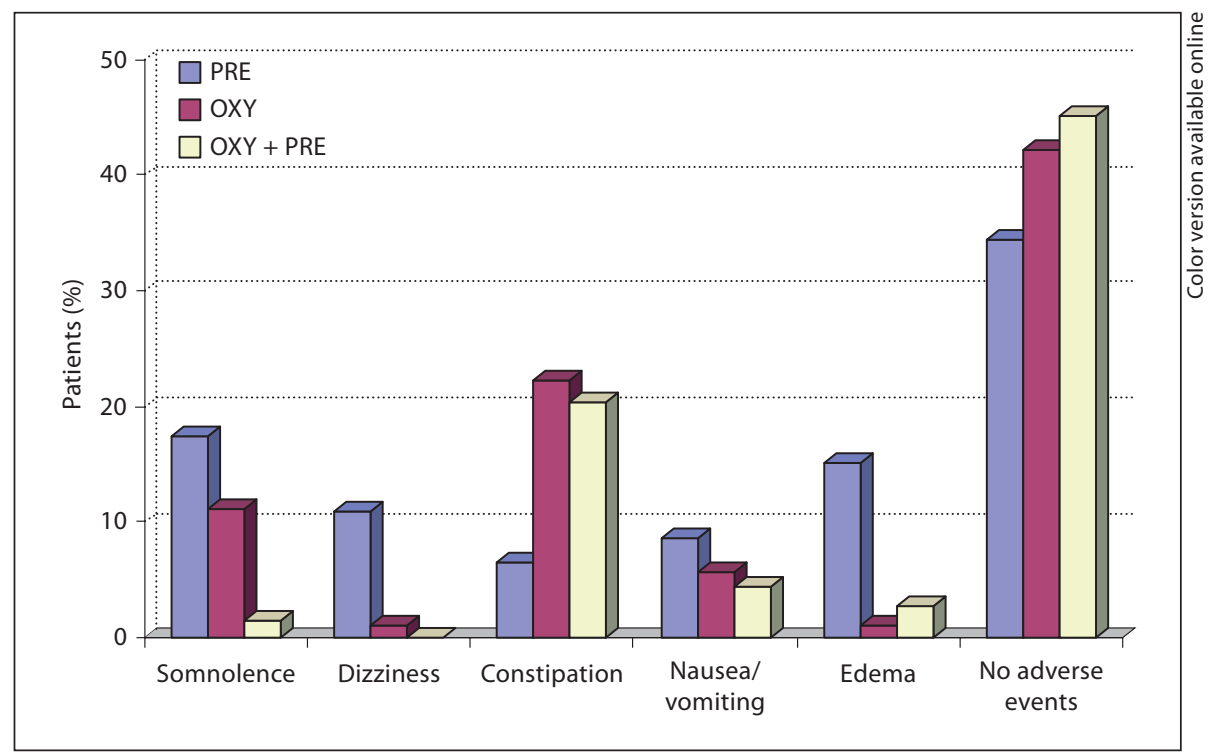

\section{Discussion}

This comparative study enrolled 409 patients with neuropathic pain of various etiologies. At baseline, pain was of moderate to severe intensity despite the fact that most patients had received analgesic treatment, including opioids and multiple-drug therapies. These patients were therefore good candidates for treatment with a strong CR opioid, as recommended by current guidelines [3, 4], and for the study of novel, potentially more potent, drug combinations.

In terms of pain relief, the efficacy of combined CR oxycodone and pregabalin was significantly better than that of pregabalin monotherapy, and was similar to CR oxycodone monotherapy. Both combination treatment and oxycodone monotherapy had substantially greater reductions in pain intensity compared with pregabalin alone ( -80 and -76 vs. $-46 \%)$. In these two groups, the decrease in pain scores from baseline to the end of treatment was $>2$ points and was therefore considered to be clinically relevant $[20,21]$. The similar efficacy observed with the two oxycodone-based treatments (either alone or in combination with pregabalin) is in agreement with the substantial opioid responsiveness for neuropathic pain reported by other studies focused on opioid-based therapies with or without concomitant analgesic treatment [15]. The results of the current study are particularly relevant in view of the baseline data which showed that at study entry pain intensity was higher in the CR oxycodone-based treatment arms.

CR Oxycodone and Pregabalin in Neuropathic Pain
As expected, other parameters of pain relief were improved by combination treatment and treatment with CR oxycodone monotherapy, compared with pregabalin monotherapy. Patients receiving CR oxycodone-based treatment had, over the course of the study, a decrease in the number of pain crises per day, most likely as a consequence of the more sustained analgesic action of CR oxycodone [13].

Treatment with the combination therapy CR oxycodone plus pregabalin meant pain relief was achieved with lower mean dosages of the two drugs, compared to the dosages used when these agents were administered as monotherapy. The pregabalin dosage was reduced by one half and that of CR oxycodone by about one fifth. These findings are particularly relevant in the management of chronic conditions, such as neuropathic pain, requiring long-term treatments with a significant potential for the development of adverse events and/or tolerance and addiction, as may be the case with opioid analgesics [24].

QoL is a consideration in the management of chronic conditions. At the end of treatment all domains considered for the assessment of QoL were favorably influenced by combination treatment. Overall, combination therapy was associated with less interference of pain with activities of daily living than when either CR oxycodone or pregabalin were administered as monotherapy. The difference between the two oxycodone-based treatments was statistically significant, with combination therapy leading to a $71.0 \%$ improvement of the BPI ratings compared with $60.7 \%$ with CR oxycodone alone.

Eur Neurol 2009;61:129-137 
Finally, there was high patient satisfaction with CR oxycodone-based treatment; at the end of the study, $>90 \%$ of patients gave positive ratings ('effective' and 'very effective') while $<20 \%$ of patients receiving pregabalin reported that treatment was effective. These results suggest that treatment compliance may well be improved with CR oxycodone-based therapy.

Overall, combination treatment had a better tolerability profile than the two monotherapy regimens. Compared with patients on monotherapy, a smaller proportion of patients on combination therapy interrupted treatment due to adverse events. Presumably, as a consequence of the substantial reduction in the dosage of pregabalin, there was a significant decrease in the frequency of the adverse events typically associated with pregabalin when it was administered in combination with CR oxycodone [25]. However, in line with published data, adverse events associated with opioid treatment, such as constipation, were more persistent [4]. Nevertheless, in patients receiving combination therapy, all adverse events tended to decrease in frequency over the course of treatment.

This study is the first to evaluate the combination of $\mathrm{CR}$ oxycodone plus pregabalin in clinical practice. The strength of this comparative analysis lies in the large patient population and the long study duration, both of which are significantly greater than the majority of published studies on opioid agonists $[3,4,26]$. The possibil- ity of controlling pain efficiently, while decreasing dosages, and therefore toxicity and costs, deserves to be investigated more in detail. Specifically, a randomized controlled study is needed to investigate whether dosages could be further reduced to improve safety, and how the combination of an opioid analgesic and a calcium channel modulator could be optimized to achieve long-term pain relief.

In conclusion, the results of this study clearly show that the combination of CR oxycodone and pregabalin improves pain and QoL of patients with chronic, moderate to severe neuropathic pain. Combination treatment allows the use of lower doses of both agents and is therefore safer. Based on these preliminary results, combination treatment with CR oxycodone plus pregabalin could represent a valuable option for the pharmacologic treatment of chronic, neuropathic pain which is nonresponsive to current first-line treatment options.

\section{Acknowledgments}

The authors would like to thank all those involved in conducting this study and in particular the following: M. Gubernari, A. Carucci, F. Della Badia, M. Di Carlo, M. Bosco, N. Lo Martire, M. Peverini, S. Laganà, A. Molfese, and M. Stefani. Editorial assistance for the preparation of this paper was provided by Anna Battershill, Wolters Kluwer Health Medical Communications.

\section{References}

1 Davis MP: What is new in neuropathic pain? Support Care Cancer 2007;15:363-372.

2 Chong MS, Hester J: Diabetic painful neuropathy. Current and future treatment options. Drugs 2007;67:569-585.

- 3 Attal N, Cruccu G, Haanpää M, Hansson P, Jensen TS, Nurmikko T, Sampaio C, Sindrup S, Wiffen P; EFNS Task Force: EFNS guidelines on pharmacological treatment of neuropathic pain. Eur J Neurol 2006;13:11531189.

- 4 Dworkin RH, O’Connor AB, Backonja M, Farrar JT, Finnerup NB, Jensen TS, Kalso EA, Loeser JD, Miaskowski C, Nurmikko TJ, Portenoy RK, Rice AS, Stacey BR, Treede RD, Turk DC, Wallace MS: Pharmacologic management of neuropathic pain: evidencebased recommendations. Pain 2007;132: 237-251.
5 Namaka M, Gramlich CR, Ruhlen D, Melanson M, Sutton I, Major J: A treatment algorithm for neuropathic pain. Clin Ther 2004; 26:951-979.

6 Gilron I, Max MB: Combination pharmacotherapy for neuropathic pain: current evidence and future directions. Expert Rev Neurother 2005;5:823-830.

7 Matthews EA, Dickenson AH: A combination of gabapentin and morphine mediates enhanced inhibitory effects on dorsal horn neuronal responses in a rat model of neuropathy. Anesthesiology 2002;96:633-640.

8 Gilron I, Bailey JM, Tu D, Holden RR, Weaver DF, Houlden RL: Morphine, gabapentin, or their combination for neuropathic pain. $\mathrm{N}$ Engl J Med 2005;352:1324-1334.

-9 Rosenstock J, Tuchman M, LaMoreaux L, Sharma U: Pregabalin for the treatment of painful diabetic peripheral neuropathy: a double-blind, placebo-controlled trial. Pain 2004;110:628-638.
10 Lesser H, Sharma U, LaMoreaux L, Poole RM: Pregabalin relieves symptoms of painful diabetic neuropathy: a randomized controlled trial. Neurology 2004;63:2104-2110.

- 11 Richter RW, Portenoy R, Sharma U, Lamoreaux L, Bockbrader H, Knapp LE: Relief of painful diabetic peripheral neuropathy with pregabalin: a randomized, placebo-controlled trial. J Pain 2005;6:253-260.

12 Freynhagen R, Strojek K, Griesing T, Whalen E, Balkenohl M: Efficacy of pregabalin in neuropathic pain evaluated in a 12-week, randomized, double-blind, multicentre, placebo-controlled trial of flexible- and fixeddose regimens. Pain 2005;115:254-263.

13 Davis MP, Varga J, Dickerson D, Walsh D, LeGrand SB, Lagman R: Normal-release and controlled-release oxycodone: pharmacokinetics, pharmacodynamics, and controversy. Support Care Cancer 2003;11:84-92. 
14 Watson CP, Babul N: Efficacy of oxycodone in neuropathic pain: a randomized trial in postherpetic neuralgia. Neurology 1998;50: 1837-1841.

15 Watson CP, Moulin D, Watt-Watson J, Gordon A, Eisenhoffer J: Controlled-release oxycodone relieves neuropathic pain: a randomized controlled trial in painful diabetic neuropathy. Pain 2003;105:71-78.

16 Wörz R, Frank M, Achenbach U: Controlledrelease oxycodone - A therapeutic option for severe neuropathic pain. Two multicenter observational studies. MMW Fortschr Med 2003;145(suppl 3):71-76.

- 17 Gimbel JS, Richards P, Portenoy RK: Controlled-release oxycodone for pain in diabetic neuropathy: a randomized controlled trial. Neurology 2003;60:927-934.
18 Kalso E, Allan L, Dellemijn PL, Faura CC, Ilias WK, Jensen TS, Perrot S, Plaghki LH, Zenz M: Recommendations for using opioids in chronic non-cancer pain. Eur J Pain 2003;7:381-386.

19 Apolone G, Mangano S, Compagnoni A, Negri E, Mosconi P, Mannino S, Villa M, Zuccaro P; Cancer Pain Outcome Research Study Group (CPORSG): A multidisciplinary project to improve the quality of cancer pain management in Italy: background, methods, and preliminary results. J Ambul Care Manage 2006;29:332-341.

20 Farrar J, Young JP Jr, LaMoreaux L, Werth JL, Poole RM: Clinical importance of changes in chronic pain intensity measured on an 11-point numerical pain rating scale. Pain 2001;94:149-158.

21 Rowbotham M: What is a clinically meaningful reduction in pain? Pain 2001;94:131132 .
22 Cleeland CS, Ryan KM: Pain assessment: global use of the Brief Pain Inventory. Ann Acad Med Singapore 1994;23:129-138.

23 Bonezzi C, Nava A, Barbieri M, Bettaglio R, Demartini L, Miotti D, Paulin L: Validazione della versione italiana del Brief Pain Inventory nei pazienti con dolore cronico. Minerva Anestesiol 2002;68:607-611.

24 Eisenberg E, McNicol ED, Carr D: Efficacy and safety of opioid agonists in the treatment of neuropathic pain of nonmalignant origin. JAMA 2005;293:3043-3052.

25 Gilron I: Gabapentin and pregabalin for chronic neuropathic and early postsurgical pain: current evidence and future directions. Curr Opin Anaesthesiol 2007;20:456-472.

26 Eisenberg E, McNicol ED, Carr DB: Efficacy of mu-opioid agonists in the treatment of evoked neuropathic pain: systematic review of randomized controlled trials. Eur J Pain 2006;10:667-676. 